\title{
L. On differential double refraction
}

\section{E.J. Rendtorff M.S.}

To cite this article: E.J. Rendtorff M.S. (1901) L. On differential double refraction, Philosophical Magazine Series 6, 1:5, 539-548, DOI: 10.1080/14786440109462642

To link to this article: http://dx.doi.org/10.1080/14786440109462642

册 Published online: 08 Jun 2010.

Submit your article to this journal $\pi$

Џll Article views: 2

Q View related articles ¿

Citing articles: 1 View citing articles $\square$ 
[ 539$]$

L. On Differential Double Refraction. By E. J. ReNDTORF, M.S., Fellow in Physics, University of Nebraska*.

TF a train of waves, polarized at a definite angle to the 1 principal axis of a crystalline plate, pass perpendicularly through the same, the retardation of the component lightwaves is a function of the thickness of the crystal, the differential dispersion, and the absolute wave-length of the light.

The object of the present investigation was to determine this relative retardation in different crystals for the different wave-lengths in the visible spectrum. The method was similar to that first used by Prof. D. B. Brace $\dagger$. This method greatly simplifies the work inasmuch as the order can be at once determined by means of the plate itself, the only requisite being that the thickness of the plate vary.

If a crystalline wedge or plate be placed under a polariscope so that the principal axis of a crystal makes an angle of $45^{\circ}$ with the plane of polarization of incident white light, the nicols being crossed, and examined with a spectroscope, a series of sharply defined black interference-bands will be seen to cross the spectrum, as first discovered by Müller. These black bands occur where

$$
\frac{\mathrm{D}\left(n_{e}-n_{0}\right)}{\lambda}=\mathrm{N}
$$

is a whole number, $n_{e}$ and $n_{0}$ being the principal indices, $\lambda$ the wave-length, and $D$ the thickness and $N$ the order of the plate. The disposition of these lines in the spectrum is dependent upon the actual differential dispersion of the crystal for different periods. As the crystal is changed in thickness the interference-bands move across the spectrum. If the differential dispersion be normal and the crystal be increased in thickness, the bands move toward the red; if diminished, toward the violet end of the spectrum. If now the number of bands $m$ passing say the $D$ line be counted$m$ being reckoned positive when the bands move toward the red-and if $n$ is the ratio of the initial to the final number of bands between any two wave-lengths, we have

whence

$$
n=\frac{\mathrm{N}}{\mathrm{N}+m},
$$

$$
\mathrm{N}=\frac{m n}{1-n}
$$

* Communicated by Prof. D. B. Brace.

+ Phil. Mag. Oct. 1899, p. 345.

$$
2 \mathrm{~N} 2
$$


which gives us the original order. (This is given with the wrong sign on p. 350 in the paper by Prof. Brace.) If $\cdot 01$ of a band can be measured this method may be used for determining the order, without any error, up to $n=25$.

For comparison of different crystals for the purpose of achromatizing different combinations of plates-for example, pairs of crystals for producing achromatic retardation-plates - adjoining interference-spectra from each plate may be used and the thickness varied until the bands coincide in any part of the spectrum which it is desired to achromatize.

Thus if

$$
\mathrm{N}^{\prime}=\mathrm{D}^{\prime} \frac{n_{e}^{\prime}-n_{0}^{\prime}}{\lambda} \quad \text { and } \quad \mathrm{N}^{\prime \prime}=\mathrm{D}^{\prime \prime} \frac{n_{e}^{\prime \prime}-n_{0}^{\prime \prime}}{\lambda}
$$

are the orders of each plate, then

$$
\frac{\delta\left(\mathrm{N}^{\prime}+\mathrm{N}^{\prime \prime}\right)}{\delta \lambda}=0, \quad \text { or } \quad \frac{\delta \mathrm{N}^{\prime}}{\delta \lambda}=-\frac{\delta \mathrm{N}^{\prime \prime}}{\delta \lambda},
$$

if there is to be achromatism for any part of the spectrum $\lambda$. The minus sign indicates that the plates are to be placed in subtractive order.

In general such a similar disposition of the bands does not obtain, but there will always be certain relative orders which give the best coincidence of these bands, either between two certain periods or throughout the entire visible spectrum.

To determine the best relative orders, the cross-hair of the observing telescope was adjusted so as to coincide with the position midway between the Na lines, and the crystalline wedges were then shifted until the best coincidences on either side of the Na lines were obtained. If now one of the wedges is shifted until the ratio of the bands between any two wavelengths is $n$, and if $m$ bands have passed the cross-hair, $m$ being taken positive or negative according to the direction of displacement, the order will be $\mathrm{N}=\frac{m n}{1-n}$ for the $\mathrm{Na}$ lines.

If the adjoining interference-spectra have the same differential dispersion and are produced by crystals, one in the shape of a wedge and the other a plane plate, an exact coincidence is always possible throughout the entire visible spectrum, and the order of the plate may be exactly determined even when this order is several thousands.

The apparatus was arranged as shown diagranmatically in fig. 1. N, N represent the Nicol prisms, which are crossed at an angle of $45^{\circ}$ to the principal axes of the crystalline wedges B, B. A, A are smaller wedges of the same crystal 
having their principal axes parallel to those of the corresponding larger wedges and the same angle, and C, C plane plates used to obtain higher orders than are possible with the wedges alone. $\mathrm{L}$ is a platform, moveable by means of the

Fig. 1.

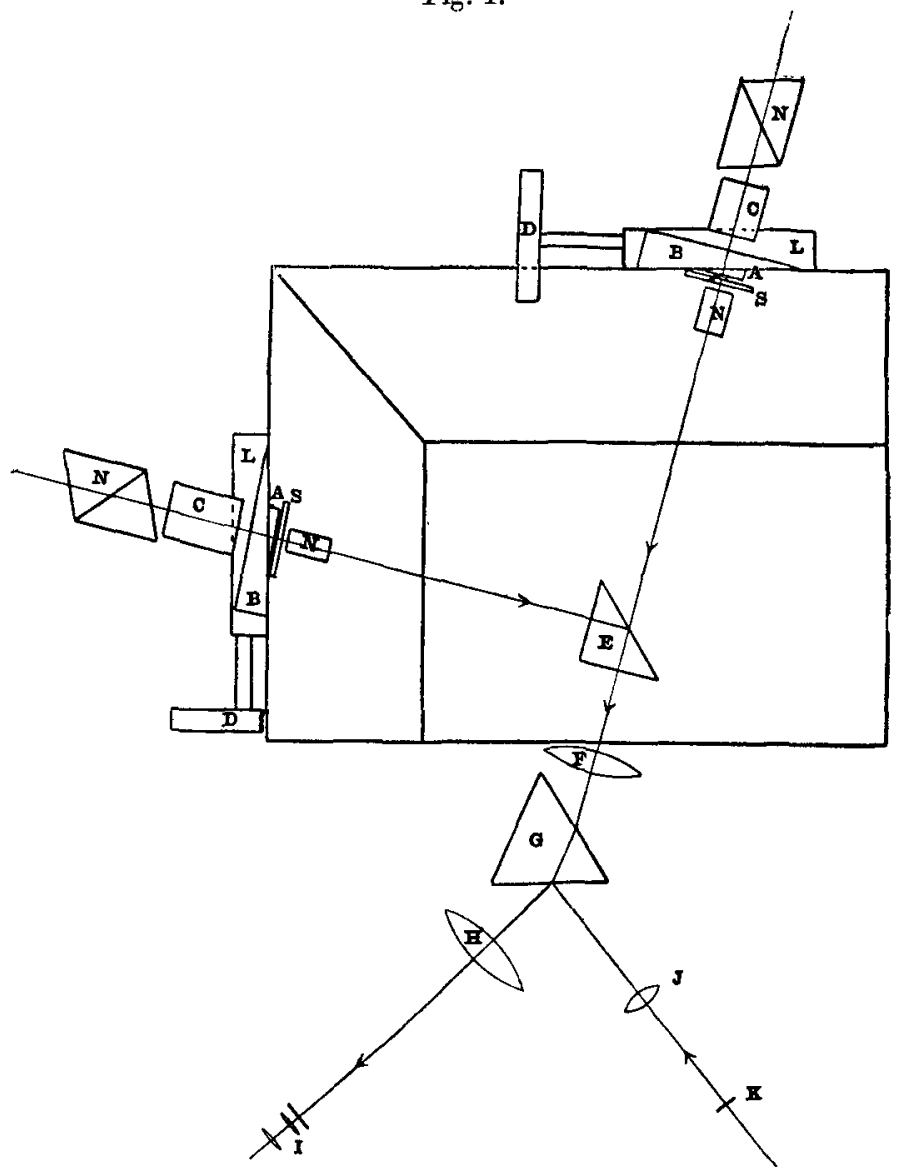

screw $\mathrm{D}$, on which the wedges $\mathrm{B}, \mathrm{B}$ are mounted. $\mathrm{E}$ is a totally-reflecting prism, $\mathrm{F}$ the collimating-lens, and $\mathrm{G}$ a flintglass prism placed at the angle of minimum deviation for Na light. $\mathrm{H} I$ is the observing-telescope, and $\mathrm{K}$ a photographic scale placed at the principal focal length from the achromatic lens J.

The collimator-slits S, S were ruled on silvered glass with a sharp knife. These slits should be close to the wedges, through which the light passes normal to their optic axes. 
The apparatus is so adjusted that the two spectra are contiguous with their Fraunhofer lines in exact coincidence, and no parallax between the interference-bands and the scale.

The wedges were now adjusted until the coincidence, at equal periods on either side of the Na line, was the best obtainable. The cross-hair was then set midway between the $\mathrm{D}_{1}$ and $\mathrm{D}_{2}$ lines, where coincident interference-bands were always formed, and the orders of the two crystals determined as previously described.

'I'he wedges and auxiliary plates were cut from crystals of Iceland spar, quartz, and of selenite and mica parallel to the principal cleavage-planes, and were of sufficient thickness to give orders of sereral bundreds or a thousand. On account of the great absorption of mica, high orders could not be used. This, however, was unnecessary in the comparison with other crystals, since its differential dispersion differs more from the crystals examined than any other, and relatively low orders are sufficient for determining the orders of achromatism. The best coincidences were obtained for the :$40^{\prime \prime}$ order for $\mathrm{Na}$ light of mica when combined with $35^{\prime \prime}$ order of selenite ;

$45^{\prime \prime}$ order for Na light of mica when combined with the 40 " order of quartz;

$222^{\prime \prime}$ order for Na light of quartz when combined with the $212^{\prime \prime}$ order of Iceland spar;

$210^{\prime \prime}$ order for Na light of selenite when combined with the $198^{\prime \prime}$ order of Iceland spar;

$606^{\prime \prime}$ order for Na light of selenite when combined with the $594^{\prime \prime}$ order of quartz.

From the orders obtained for mica when combined with quartz and with selenite respectively, it follows that the $64^{\prime \prime}$ order of quartz, for Na light, produces the best achromatism when combined with the $63^{\prime \prime}$ order of selenite. These relative orders give a fair coincidence of bands in the red, but leave the green uncompensated. The orders 101 selenite to 99 quartz give a far better compensation throughout the visible spectrum, especially between the yellow and blue, but achromatize poorly in the red. With this combination of crystals, as also with selenite and Iceland spar, it is possible to achromatize for certain periods only. Mica and selenite, as well as Iceland spar and quartz, give a good coincidence of bands throughout the entire spectrum.

In order to study the disposition of the interference-bands with respect to the wave-length for various orders of selenite and quartz, the refracting-prism G, fig. 1, was replaced by a plane grating. By means of an achromatic lens $\mathrm{K}$, fig. 2, 
a scale, M, graduated in fifth millimetres, was projected into the telescope, H I, and the position of the bands plotted. The setting of the grating in order to reflect the scale into the eyepiece prevented the formation of a strictly normal

Fig. 2.

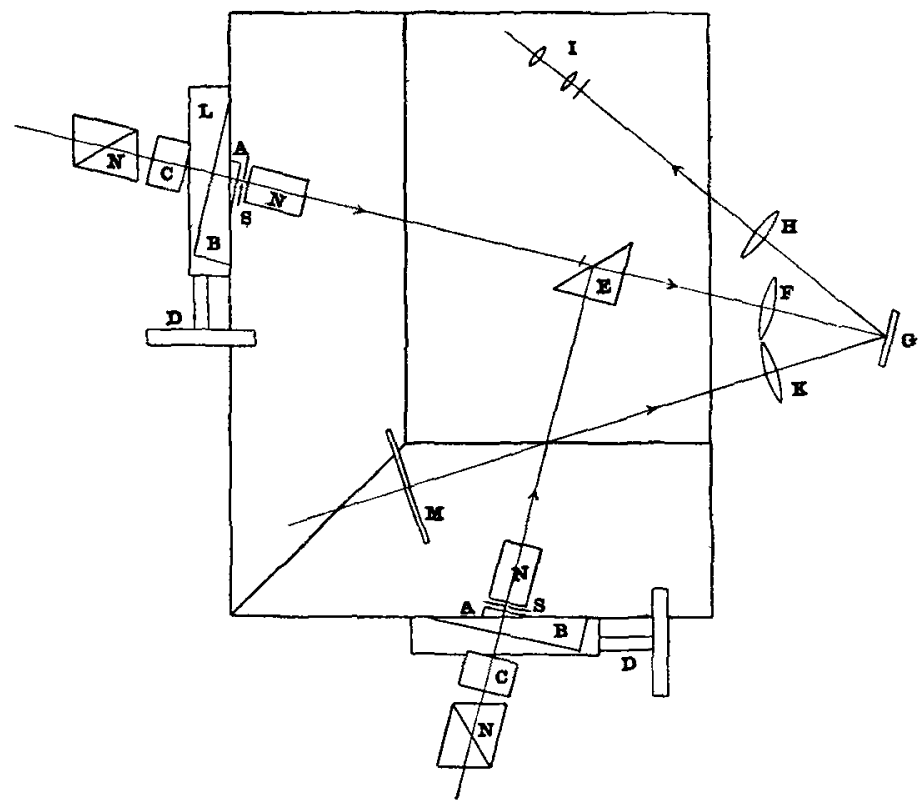

spectrum, but the spectrum observed was sufficiently so to indicate the true character of the distribution of the bands. Fig. 3 shows different relative positions of the bands in selenite and quartz in the spectrum. The first system shows that the orders 65 selenite and 66 quartz coincide better in the orange and red than the third system of 67 selenite and 66 quartz; but the latter coincide much better in the green and blue. The last named orders give a good coincidence between yellow and blue where the light is intense. The systems show that in the normal spectrum the bands are furthest apart in the red and narrowest in the blue. This agrees with the theory of the distribution of these bands as given by Prof. Brace. The curves in fig. 4 indicate the relation of the order to the deviation in the irrational refraction-spectrum, the image of the scale representing abscissæand the number of the black interferencebands the ordinates. The band coinciding with the $\mathrm{Na}$ line is represented as the zero band. The orders used were 75 for mica, 88.5 for Iceland spar, 187 for selenite, and 190 for quartz. 
Fig. 3.
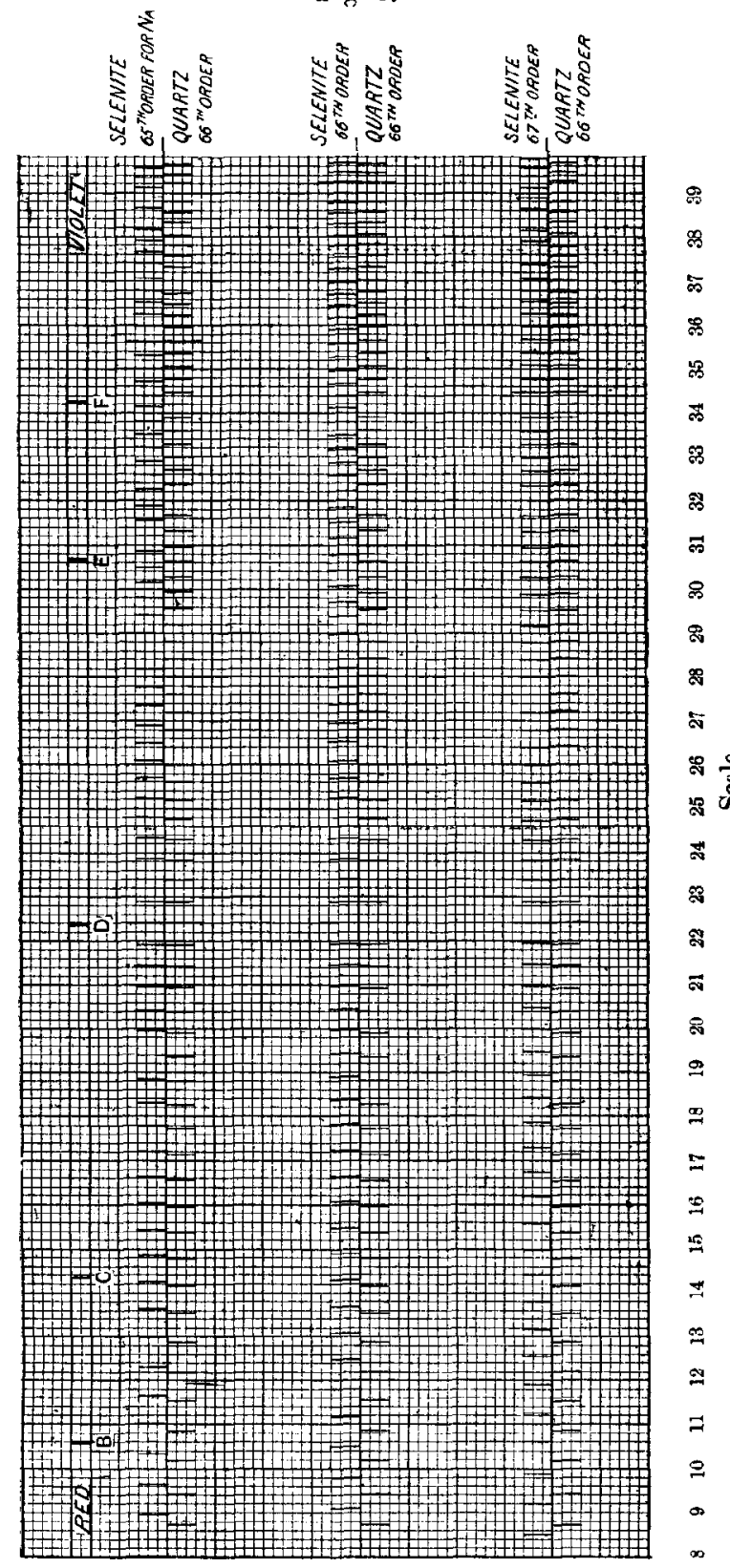
Differential Double Refiaction.

Fig. 4.

ORDINATES FOF. SELENITE ANO QUARTZ

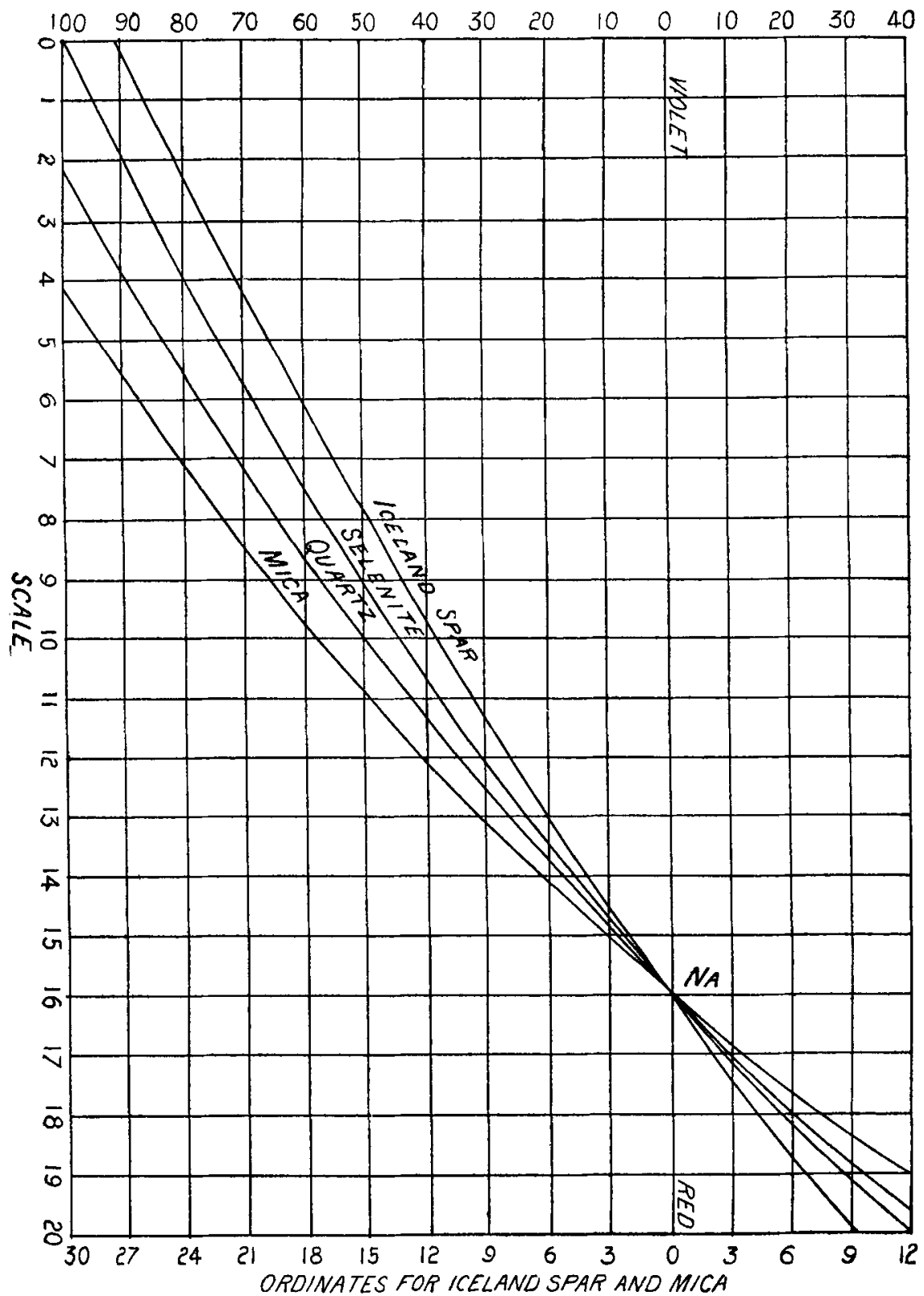


To test for achromatism in a crossed pair of plates, let us assume that two crystals of the orders $N_{1}$ and $N_{2}$ present a maximum achromatism. If crystal plates of these, of the orders $\frac{N_{1}}{4\left(N_{1}-N_{2}\right)}$ and $\frac{N_{2}}{4\left(N_{1}-N_{2}\right)}$, be crossed, an achromatic quarter wave-plate results. The orders 2 of mica and $1 \frac{3}{4}$ of selenite, for $\mathrm{Na}$ light, were used for one of these pairs of plates. A Fresnel rhomb was placed at an angle of $45^{\circ}$ to the plane of polarization of the polarizer, and, with nicols crossed, the achromatic plate was placed above the rhomb in such a position as to give a minimum illumination. The light passing through the analyser was then examined with a spectroscope. Total darkness existed in the neighbourhood of the Na line, with a gradual increase of illumination toward the red and violet, showing that the colours had not been compensated for perfectly. When placed under a polariscope the achromatic plate showed very little change of tint on revolving the analyser. It consequently approximated to the ideal achromatic condition.

Let us assume that we have two crystals, such as Iceland spar and quartz, of the $212^{\prime \prime}$ and $222^{\prime \prime}$ order for $\mathrm{Na}$ light, respectively. Let us furthermore assume that the interference-bands produced by them coincide at the $\mathrm{Na}$ lines, and that the last visible red band in the quartz extends $\frac{1}{10}$ of a band beyond the corresponding one in selenite, while a certain violet band in the selenite spectrum extends a similar distance beyond the corresponding quartz band. Orders of 5.55 for quartz and 5.30 for selenite will then make a plate whose phase-retardations will be $90^{\circ} 25^{\prime}$ for the extreme red, $90^{\circ}$ for the yellow, and $89^{\circ} 35^{\prime}$ for the violet rays.

In conclusion, the author desires to thank Professor Brace for many valuable suggestions that enabled him to carry out this work.

[Note.-In determining the order of crystal-plates observers, as well as cutters, generally have committed serious errors, at least for the higher orders; and the tabulated indices must consequently be erroneous to some extent. In this method of determining the order no error is possible ordinarily, it being merely in matter of counting. The method most generally used is to determine the order by comparison with a plate or wedge of another crystal, usually of quartz. As already shown, this method cannot be used directly without knowing the relative orders for achromatism of the two crystals, and correcting accordingly. Heretofore the number 
of bands for homogeneous light between the zero order and the position of the white band, resulting from the plate to be compared and the plate of reference, has been taken directly as the order of the unknown plate.

An example of such an error probably occurs in Dufet's * determination of the optical constants of selenite. Instead of nsing a comparison wedge, as usually done, he used as referenceplate Iceland spar cut normal to the optic axis and placed between the polarizer and analyser of a polarizing-microscope so as to obtain the characteristic rings and black cross. The selenite plate being placed next to this and adjusted until the rings are visible, the position of the black ring is noted, and then the order of the ring determined by substituting sodium light. His apparent orders vary for plates of different thickness from $27 \cdot 28$ up to $83 \cdot 37$. Now from the results obtained above, the order 33 of Iceland spar corresponds to the order 35 of selenite (parallel to the principal cleavage-plane). Hence, assuming the differential dispersion of Iceland spar to be the same for different directions and neglecting the effect of oblique transmission, the order 27 ought to be 28 , or more probably 29 , and 83 should be 88 for selenite; that is, the difference between the greatest and least indices should be increased by approximately 6 per cent., or from 00918 to $\cdot 00973$. Now the observations of von Lang $\uparrow$ by means of a prism give $\cdot 009665$ for selenite. Mouton $\ddagger$ obtained by means of interferencebands $\cdot 00988$. The order of his plate he determined by the method of successive approximations, using the first two terms in Cauchy's formula. Dufet, however, attributes an error of one order too much in Mouton's plate, and then finds results concordant with his own. The astonishing agreement between results of Dufet with a prism and with a plate should be mentioned. However, that the orders of his plates are seriously in error cannot be doubted, as he mentions no correction. The apparent anomalous differential dispersion obtained by Mouton is not confirmed by Dufet. The results obtained above do not indicate any such state. Compare figs. 3 and 4 . It would be desirable to have the correction referred to introduced into the various tabular results given for crystals by different observers, or new data obtained with these corrections. Work of this nature is now in progress in this laboratory. This process of obtaining the order of interference-bands has been extended to the determinations of indices by interference methods, comparisons being made

* Dufet, Journ. de Physique (2) vii. p. 297 (1888).

I Wien. Ak. Ber. xxxvi. (2) p. 793 (1877).

$\ddagger$ Compt. Rend. Ixxxviii. p. 987 (1879). 
directly with the bands obtained in vacuo or in air for the same path. The results of experiments now in progress demonstrate the greater simplicity and accuracy of the method as compared with others.-D. B. BRACE.]

Physical Laboratory,

University of Nebraska, Lincoln.

\section{Note on the Double Refraction of Electric Waves.} By George Pierce, Ph.D.*

TN the February number of the Philosophical Magazine I have described an experiment on the measurement of the refractive indices for electric waves of a number of doublyrefractive media: namely, various woods. These media were all also doubly-absorptive.

In the present brief note I wish to examine the question as to whether, on Maxwell's theory, the double absorption by these media is sufficient to account for the double refraction; and, indeed, whether both of these properties can be ascribed merely to differences of conductivity of the woods along and across the grain. The treatment that follows, except the application, is taken largely from Boltzmann's Vorlesungen über Mawuells Theorie, \$96 (Leipzig, 1891).

Let $f, g, h$ be the components of electric polarization; then in a conducting medinm, of conductivity $\mathrm{C}$, the equations of the field are (Boltzmann eq. 81)

$$
\mu k \frac{d^{2} f}{d t^{2}}+4 \pi \mu \mathrm{C} \frac{d f}{d t}=\frac{d^{2} f^{2}}{d x^{2}}+\frac{d^{2} f}{d y^{2}}+\frac{d^{2} f}{d z^{2}}, \quad .
$$

and similar equations for $g$ and $h$.

Now let us assume a plane wave propagated in the direction of the Z-axis, and let the electric displacement be in the direction of the X-axis ; then $g=h=0$, and $f$ is a function of $t$ and $z$ alone; whence equation (1) becomes

$$
\mu k \frac{d^{2} f}{d t^{2}}+4 \pi \mu \mathrm{C} \frac{d f}{d t}=\frac{d^{2} f}{d \varepsilon^{2}} . \quad . \quad . .
$$

A particular solution of (2) is

$$
f=\mathrm{B} e^{\frac{2 \pi}{T} t i+\left(\xi+r_{i}\right) z}
$$

where $\mathrm{B}, \mathrm{T}, \xi$. and $\eta$ are constants, to determine which we

$$
\text { * Communicated by the Author. }
$$

